\title{
Mandibular advancement appliances for treating sleep apnoea/hypopnoea syndrome
}

\author{
Abstracted from \\ Zhou J and Liu YH. \\ A randomised titrated crossover study comparing two oral appliances in the \\ treatment for mild to moderate obstructive sleep apnoea/hypopnoea syndrome. \\ J Oral Rehab 2012; 39: 914-922. \\ Address for correspondence: Liu Yuehua, YanChang Middle Road NO. 399, \\ Zhabei District, Shanghai, China. E-mail: liuyuehua@tongji.edu.cn
}

Question: What is the relative efficacy of two different types of mandibular advancement appliances (MAAs) for subjective and objective parameters in adults diagnosed with mild to moderate obstructive sleep apnoea/hypopnoea syndrome (OSAHS)?

Study design Randomised controlled crossover trial. Intervention Sixteen participants (13 men and three women) with mild to moderate OSAHS, diagnosed using polysomnography (PSG), were recruited from a sleep clinic. To be included, participants had to have $\mathbf{2 0}$ or more teeth (sufficient to provide retention for oral appliances), have had no previous OSAHS treatment, been unable to tolerate nasal continuous positive airway pressure and be free from caries, periodontal disease, temporomandibular joint (TMJ) pain or movement limitations. One-night mandibular advancement titration was carried out using a custom made temporary dental appliance to reach each individual's pre-determined maximum voluntary advancement value. Participants were given one week to acclimatise. Two different MAAs were made to this titrated value for each participant; the SILENT NITE (a two-piece MAA in flexible trays connected by two plastic bars to maintain mandibular advancement) and a one-piece resin-made activator appliance which was retained by posterior tooth clasps. Patients were 'randomly divided' into two groups and wore one or other appliance as allocated for three months. Following a two week washout period, they then wore the other appliance for a further three months.

Outcome measure Subjective measures were recorded pre- and post treatment using Epworth's Sleepiness Scale and an unspecified Snoring Scale. Preferences, compliance and side effects were assessed through interviews with patients and their families. Pre- and post treatment objective measures were taken using a variety of PSG results which were manually scored by a blinded operator (these included EOG, ECG, supplemental EMG, nasal-oral airflow, abdominal and chest respiratory effort, ECG rhythm). Cephalometric radiographs taken pre- and post treatment were scored by a single examiner for upper airway space diameters.

Results Baseline measures for both BMI and titrated distances for mandibular advancement were similar for both groups. Daytime performance (ESS) and snoring (SS) showed significant improvement for both MAAs during treatment but there were no significant differences between the appliances. Out of the 16 participants, seven preferred the monoblock appliance, two preferred the two-piece and seven had no preference. All patients finished treatment and stated full compliance despite some of the participants experiencing side effects; two had ulcers for several weeks at the start of treatment, four experienced TMJ pain, three complained of muscle discomfort and four felt some dental discomfort. PSG showed that total sleep time did not differ pre- and post treatment and sleep efficiency increased for the monoblock appliance only. Both appliances showed improvement in $\mathrm{AHI}, \mathrm{Al}$ and Hypopnoea Index, but the monoblock had a more statistically important improvement for $\mathrm{AHI}$ and Al. Upper airway space diameters were increased with both MAAs but there were no significant differences between the appliances.

Conclusions Both the monoblock and the SILENT NITE appliances were effective at reducing the severity of symptoms of OSAHS. The monoblock, however, offered some advantages over the two-piece appliance in achieving a greater improvement in Apnoea Hypopnoea Index and Apnoea Index and being preferred by most patients.

\section{Commentary}

Obstructive Sleep Aponea/Hypopnoea Syndrome (OSAHS) is caused by multiple episodes of intermittent upper airways obstruction during sleep. The result of these multiple episodes of obstruction is sleep fragmentation leading to increased daytime sleepiness. This can have a significant impact on alertness, general wellbeing and has been shown to have adverse long-term health effects. Continuous positive airway pressure can be an effective treatment option but is a highly disruptive to peoples' lives and can be difficult to tolerate. Mandibular Advancement Appliances (MAAs) have been used effectively in the management of OSAHS ${ }^{1}$ and are easier to tolerate, but their effectiveness for different levels of OSAHS and the best design for MAAs are unclear.

This study compared two types of MAA, using a randomised crossover study design. Sixteen patients, selected from 65 patients who attended the Sleep Laboratory, Tongji University, participated in the trial.

Shortcomings in reporting made it difficult to establish what the primary outcome of the study was, and no sample size calculation was provided. There was no mention of how the patients were selected from the 65 , nor the randomisation technique used.

The authors used the Epworth's Sleepiness Scale (ESS) and the Snoring Scale (SS), but fail to define which snoring scale they have used. Scoring for these was by the participant's partner/family members. Participants' upper airway spaces were assessed using lateral 


\section{ORTHODONTICS, PROSTHETIC DENTISTRY}

cephalograms, pre- and post treatment. Both types of appliance significantly increased airway space, with no difference between the two designs. However, lateral cephalograms only assess airway size in two dimensions and it is a three dimensional structure, so it may have been that there were undetected differences.

The Apnoea Hypopnoea Index (AHI) subdivides OSAHS into varying degrees of abnormality (AHI score of 5-14/hr being mild; 15-30/ $\mathrm{hr}$ moderate; $>30 / \mathrm{hr}$ is severe). The authors' success criteria for a MMA was a reduction in Apnoea Hypopnoea Index (AHI) of more than $50 \%$ or an overall score less than ten. Regarding adverse events reported for 11 participants for TMJ pain, muscle discomfort, and dental discomfort, it was not specified which appliance type these events related to.

Clearly stating their primary outcome measures earlier in the paper and how the sleep efficiency was determined using the PSG measures would have aided the reader in interpretation of the clinical importance of subsequent results.

Both the monoblock and two-piece MAAs were effective in the management of mild to moderate OSAHS for the subjective measures of daytime performance and snoring, resulting in score reductions that seem clinically significant. Similarly, both MAAs were effective for the objective PSG results of AHI AI and HI. There were no significant differences between the appliances for these outcomes although the monoblock showed a statistically greater improvement for AHI and AI. However, the clinical significance of this difference was not clear.

Both MAAs resulted in clinically meaningful improvements for sleep clinic patients diagnosed with mild to moderate OHAHS and the monoblock offered some advantages over the two-piece appliance for the patients.

Dentists may have a role to play in making MAAs for patients suffering from OHAHS. However, they should liaise with medical colleagues when considering using MAA devices to alleviate symptoms to ensure that a correct diagnosis has been made and adequate subsequent monitoring is being provided.

Felicity Borrie, Alex Keightley, Suzanne Blacker and Paul Serrant Dundee Dental School, Dundee, Scotland, UK

1. Scottish Intercollegiate Guidelines Network. No 73 Management of Obstructive Sleep Apnoea/Hypopnoea Syndrome in Adults. A national clinical guideline.

Evidence-Based Dentistry (2013) 14, 27-28. doi:10.1038/sj.ebd.6400921 